\title{
Prognostic Nomogram for patients undergoing radical Pancreaticoduodenectomy for adenocarcinoma of the pancreatic head
}

\author{
Chao $\mathrm{Wu}^{\dagger}$, Sheng Zhong Hou${ }^{\dagger}$, Zuowei Wu, Xing Huang, Zihe Wang and Bole Tian*
}

\begin{abstract}
Background: Radical pancreaticoduodenectomy is the most common treatment strategy for patients diagnosed with adenocarcinoma of the pancreatic head. Few studies have reported the clinical characteristics and treatment efficacies of patients undergoing radical pancreaticoduodenectomy for adenocarcinoma of the pancreatic head.

Methods: A total of 177 pancreatic head cancer patients who underwent radical pancreaticoduodenectomy and were pathologically confirmed as having pancreatic ductal adenocarcinoma were screened in the West China Hospital of Sichuan University. The multivariate analysis results were implemented to construct a nomogram. The concordance index (c-index), the area under the curve (AUC) and calibration were utilized to evaluate the predictive performance of the nomogram.

Results: The prognostic nutritional index (PNI), the lymph node ratio (LNR) and the American Joint Committee on Cancer (AJCC) staging served as independent prognostic factors and were used to construct the nomogram. The cindexes of the nomogram were 0.799 (confidence interval $(\mathrm{Cl}), 0.741-0.858)$ and $0.732(0.657-0.807)$ in the primary set and validation set, respectively. The AUCs of the nomogram at 1 and 3 years were 0.832 and 0.783 , which were superior to the AJCC staging values of 0.759 and 0.705 , respectively.

Conclusions: The nomogram may be used to predict the prognosis of radical resection for adenocarcinoma of the pancreatic head. These findings may represent an effective model for the developing an optimal therapeutic schedule for malnourished patients who need early effective nutritional intervention and may promote the treatment efficacy of resectable adenocarcinoma of the pancreatic head.
\end{abstract}

Keywords: Adenocarcinoma of the pancreatic head, Pancreaticoduodenectomy, Prognostic, Nomogram

\footnotetext{
*Correspondence: bole_tian@163.com

${ }^{+}$Chao Wu and Sheng Zhong Hou contributed equally to this work.

Department of Pancreatic Surgery, West China Hospital, Sichuan University,

No. 37 Guoxue Alley, Chengdu, Sichuan Province, China
}

(c) The Author(s). 2021 Open Access This article is licensed under a Creative Commons Attribution 4.0 International License, which permits use, sharing, adaptation, distribution and reproduction in any medium or format, as long as you give appropriate credit to the original author(s) and the source, provide a link to the Creative Commons licence, and indicate if changes were made. The images or other third party material in this article are included in the article's Creative Commons licence, unless indicated otherwise in a credit line to the material. If material is not included in the article's Creative Commons licence and your intended use is not permitted by statutory regulation or exceeds the permitted use, you will need to obtain permission directly from the copyright holder. To view a copy of this licence, visit http://creativecommons.org/licenses/by/4.0/ The Creative Commons Public Domain Dedication waiver (http://creativecommons.org/publicdomain/zero/1.0/) applies to the data made available in this article, unless otherwise stated in a credit line to the data. 


\section{Background}

Pancreatic cancer is an extremely aggressive malignancy and has a poor prognosis worldwide [1]. Although surgical resection is a therapy implemented to treat pancreatic cancer, the rates of mortality remain high, and the 5 -year survival rate is only $10-20 \%[2$, $3]$. Consequently, it is vital to discern a postoperative prognostic biomarker that could assess the risk

Table 1 Univariate and multivariate analysis of overall survival in Primary set.

\begin{tabular}{|c|c|c|c|c|c|c|}
\hline \multirow[t]{2}{*}{ Variables } & \multirow[b]{2}{*}{ HR } & \multicolumn{3}{|c|}{ Univariate analysis } & \multicolumn{2}{|c|}{ Multivariate analysis } \\
\hline & & $95 \% \mathrm{CI}$ & P-value & HR & $95 \% \mathrm{CI}$ & P-value \\
\hline \multicolumn{7}{|c|}{ CA19-9(u/ml) } \\
\hline \multicolumn{7}{|l|}{$\leq 37$} \\
\hline (37 222] & 1.191 & $0.569-2.489$ & 0.643 & & & \\
\hline$>222$ & 1.322 & $0.642-2.714$ & 0.451 & & & \\
\hline \multicolumn{7}{|l|}{$\mathrm{CEA}(\mathrm{ng} / \mathrm{ml})$} \\
\hline \multicolumn{7}{|l|}{$\leq 5$} \\
\hline$>5$ & 1.441 & $0.785-2.644$ & 0.238 & & & \\
\hline $\operatorname{Tbil}(\mu \mathrm{mol} / \mathrm{L}$ & & & 0.693 & & & \\
\hline \multicolumn{7}{|l|}{$\leq 37$} \\
\hline$\left(\begin{array}{lll}37 & 222\end{array}\right]$ & 2.084 & $0.613-7.085$ & 0.240 & & & \\
\hline$(222-296]$ & 1.921 & $0.562-6.568$ & 0.298 & & & \\
\hline$>296$ & 2.139 & $0.511-8.957$ & 0.298 & & & \\
\hline \multicolumn{7}{|l|}{ Age } \\
\hline \multicolumn{7}{|l|}{$\leq 60$} \\
\hline$>60$ & 1.248 & $0.684-2.276$ & 0.47 & & & \\
\hline \multicolumn{7}{|c|}{ Chemotherapy } \\
\hline \multicolumn{7}{|l|}{ No } \\
\hline Yes & 0.527 & $0.187-1.481$ & 0.224 & & & \\
\hline \multicolumn{7}{|l|}{ Sex } \\
\hline \multicolumn{7}{|l|}{ Female } \\
\hline Male & 1.318 & $0.709-2.450$ & 0.383 & & & \\
\hline TNM8th & & & & 1.948 & $1.351-2.810$ & $<0.001$ \\
\hline \multicolumn{7}{|l|}{$\mathrm{I}+\mathrm{II}$} \\
\hline III & 2.403 & $1.717-3.364$ & $<0.001$ & & & \\
\hline LNR & & & & 2.543 & $1.052-6.148$ & 0.038 \\
\hline \multicolumn{7}{|l|}{$\leq 0.074$} \\
\hline$>0.074$ & 4.797 & $2.199-10.466$ & $<0.001$ & & & \\
\hline PNI & & & & 0.51 & $0.273-0.952$ & 0.034 \\
\hline \multicolumn{7}{|l|}{$\leq 48.5$} \\
\hline$>48.5$ & 0.44 & $0.239-0.812$ & 0.009 & & & \\
\hline
\end{tabular}


Table 2 Univariate and multivariate analysis of overall survival in Validation set.

\begin{tabular}{|c|c|c|c|c|c|c|}
\hline Variables & & Univariate analysis & & & Multivariate analysis & \\
\hline & HR & $95 \% \mathrm{CI}$ & P-value & HR & $95 \% \mathrm{CI}$ & P-value \\
\hline \multicolumn{7}{|c|}{ CA19-9(u/ml) } \\
\hline \multicolumn{7}{|l|}{$\leq 37$} \\
\hline (37 222] & 0.384 & $0.117-1.260$ & 0.114 & & & \\
\hline$>222$ & 0.799 & $0.437-1.460$ & 0.465 & & & \\
\hline \multicolumn{7}{|c|}{ CEA(ng/ml) } \\
\hline \multicolumn{7}{|l|}{$\leq 5$} \\
\hline$>5$ & 1.293 & $0.727-2.300$ & 0.382 & & & \\
\hline \multicolumn{7}{|c|}{$\operatorname{Tbil}(\mu \mathrm{mol} / \mathrm{L})$} \\
\hline \multicolumn{7}{|l|}{$\leq 37$} \\
\hline (37 222] & 0.816 & $0.331-2.015$ & 0.66 & & & \\
\hline$(222-296]$ & 0.642 & $0.226-1.826$ & 0.406 & & & \\
\hline$>296$ & 0.851 & $0.305-2.374$ & 0.758 & & & \\
\hline \multicolumn{7}{|l|}{ Age } \\
\hline \multicolumn{7}{|l|}{$\leq 60$} \\
\hline$>60$ & 1.264 & $0.135-2.229$ & 0.585 & & & \\
\hline \multicolumn{7}{|c|}{ Chemotherapy } \\
\hline \multicolumn{7}{|l|}{ No } \\
\hline Yes & 1.032 & $0.369-2.887$ & 0.952 & & & \\
\hline \multicolumn{7}{|l|}{ Sex } \\
\hline \multicolumn{7}{|l|}{ Female } \\
\hline Male & 1.485 & $0.836-2.638$ & 0.177 & & & \\
\hline TNM8th & & & & 2.189 & $1.493-3.211$ & $<0.001$ \\
\hline \multicolumn{7}{|l|}{$\mathrm{I}+\mathrm{II}$} \\
\hline III & 2.786 & $1.939-4.003$ & $<0.001$ & & & \\
\hline LNR & & & & 2.301 & $1.026-5.161$ & 0.043 \\
\hline \multicolumn{7}{|l|}{$\leq 0.074$} \\
\hline$>0.074$ & 4.087 & $2.065-8.090$ & $<0.001$ & & & \\
\hline PNI & & & & 0.507 & $0.273-.941$ & 0.031 \\
\hline \multicolumn{7}{|l|}{$\leq 48.5$} \\
\hline$>48.5$ & 0.398 & $0.217-0.729$ & 0.003 & & & \\
\hline
\end{tabular}

stratification of patients and help develop an optimal therapeutic schedule. Some studies have disclosed clinical characteristics, such as resection margins, the PNI, the LNR, portal vein invasion and tumor differentiation, utilized to discriminate treatment outcomes in patients with pancreatic cancer [4-8]. Additionally, only a single indicator was used to assess postoperative survival for pancreatic cancer in those 

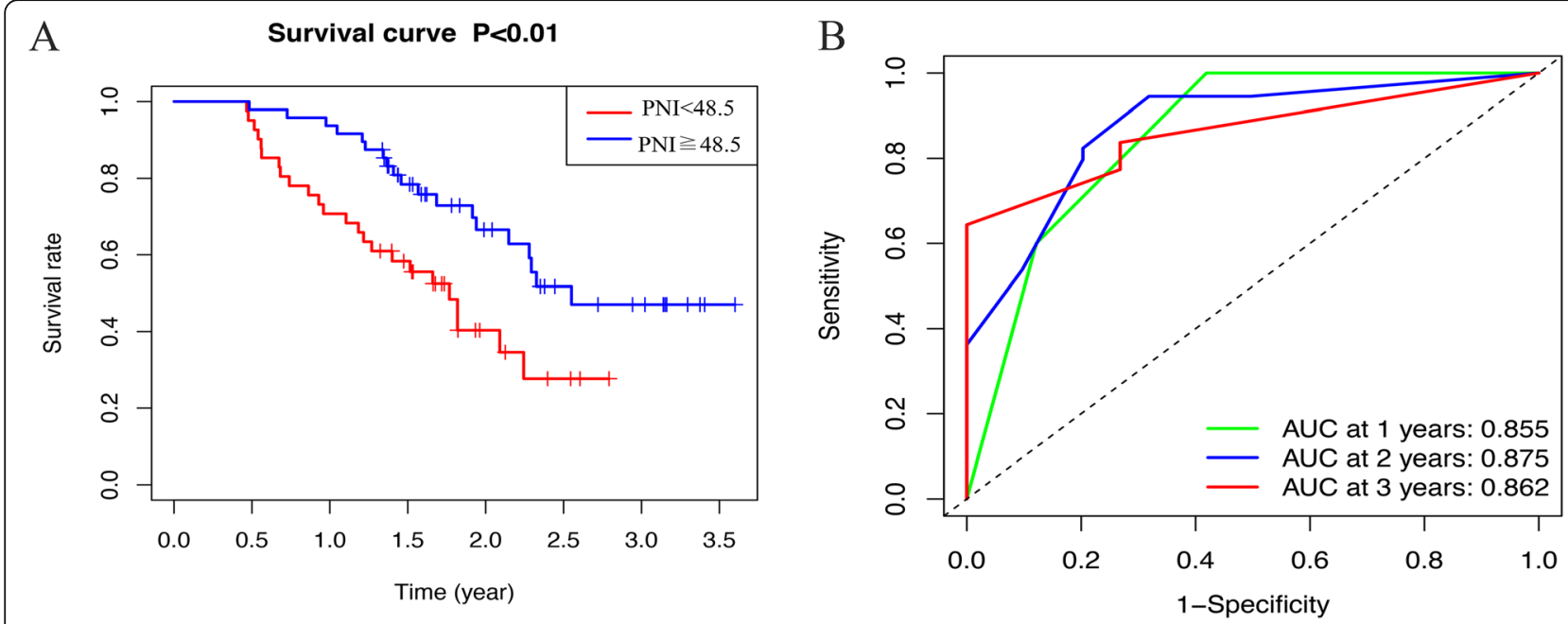

$\mathrm{C}$

$\mathrm{D}$
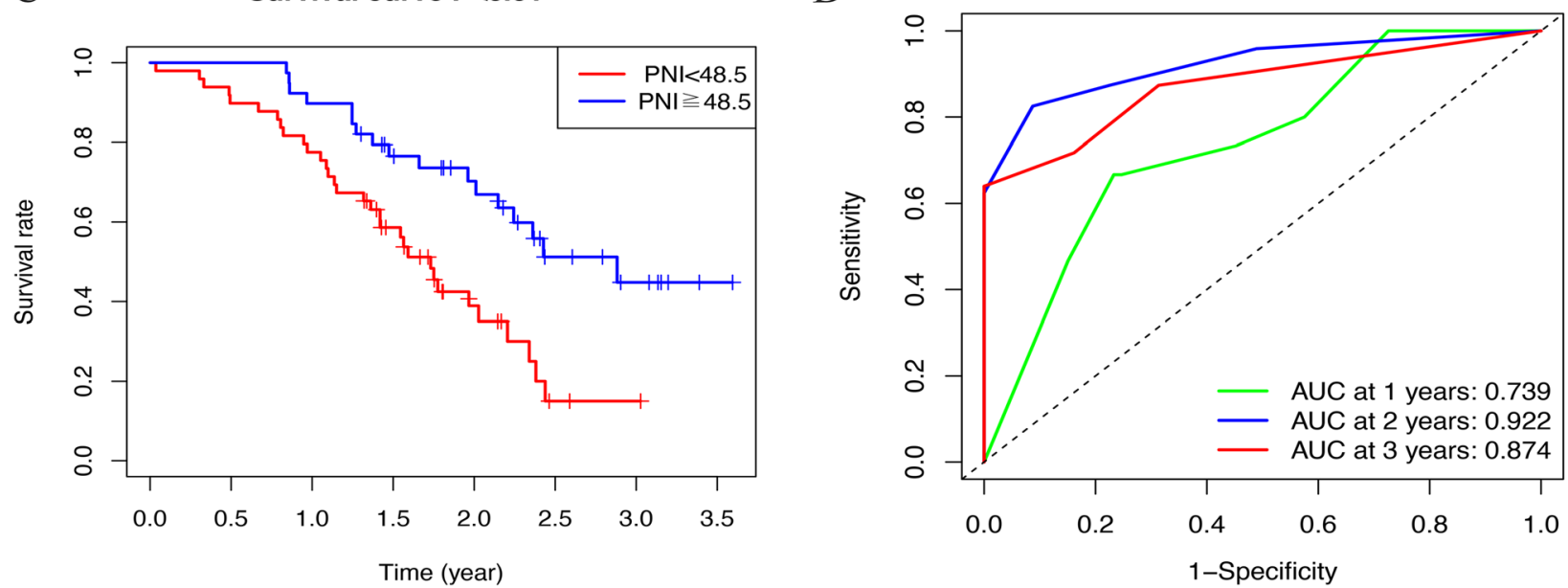

Fig. 1 The Kaplan-Meier analysis and performance of PNI in predicting 1-and 3-year prognosis respectively in primary set (ab) and validation set (cd)

studies. For this reason, we discern some indicators that serve as prognostic markers that influence the postoperative outcome of pancreatic cancer.

The prognostic nutritional index (PNI) was first identified as a prognostic marker for patients with gastrointestinal cancer and calculated from the serum albumin level and total lymphocyte count [9]. Kanda and colleagues disclosed that the PNI was associated with overall survival (OS) and that it may be a predictor with moderate accuracy in resectable pancreatic cancer. Additionally, Lee and colleagues revealed that the PNI may be a prognostic marker for all stages of pancreatic cancer [10]. These studies found that the PNI is expected to act as a surrogate marker for preoperative assessments of the nutritional and immunological status.

The lymph node ratio (LNR) is the ratio of the number of positive lymph nodes to the total number of lymph nodes dissected during surgery [11, 12].
Previous studies have unveiled that the LNR may be a sensitive indicator of OS in patients with pancreatic cancer [13-16]. The aim of this work is to evaluate the prognostic influence of the LNR and PNI on survival in patients with adenocarcinoma of the pancreatic head undergoing radical pancreaticoduodenectomy.

\section{Methods \\ Patients}

This study was approved by the Ethical Review Committees of Sichuan University and was performed in accordance with the ethical standards and according to the Declaration of Helsinki. We retrospectively collected data from 316 patients with supposed pancreatic head cancer who were admitted to the West China Hospital of Sichuan from July 2014 to June 2017. Patients with any of the following characteristics were excluded from this 


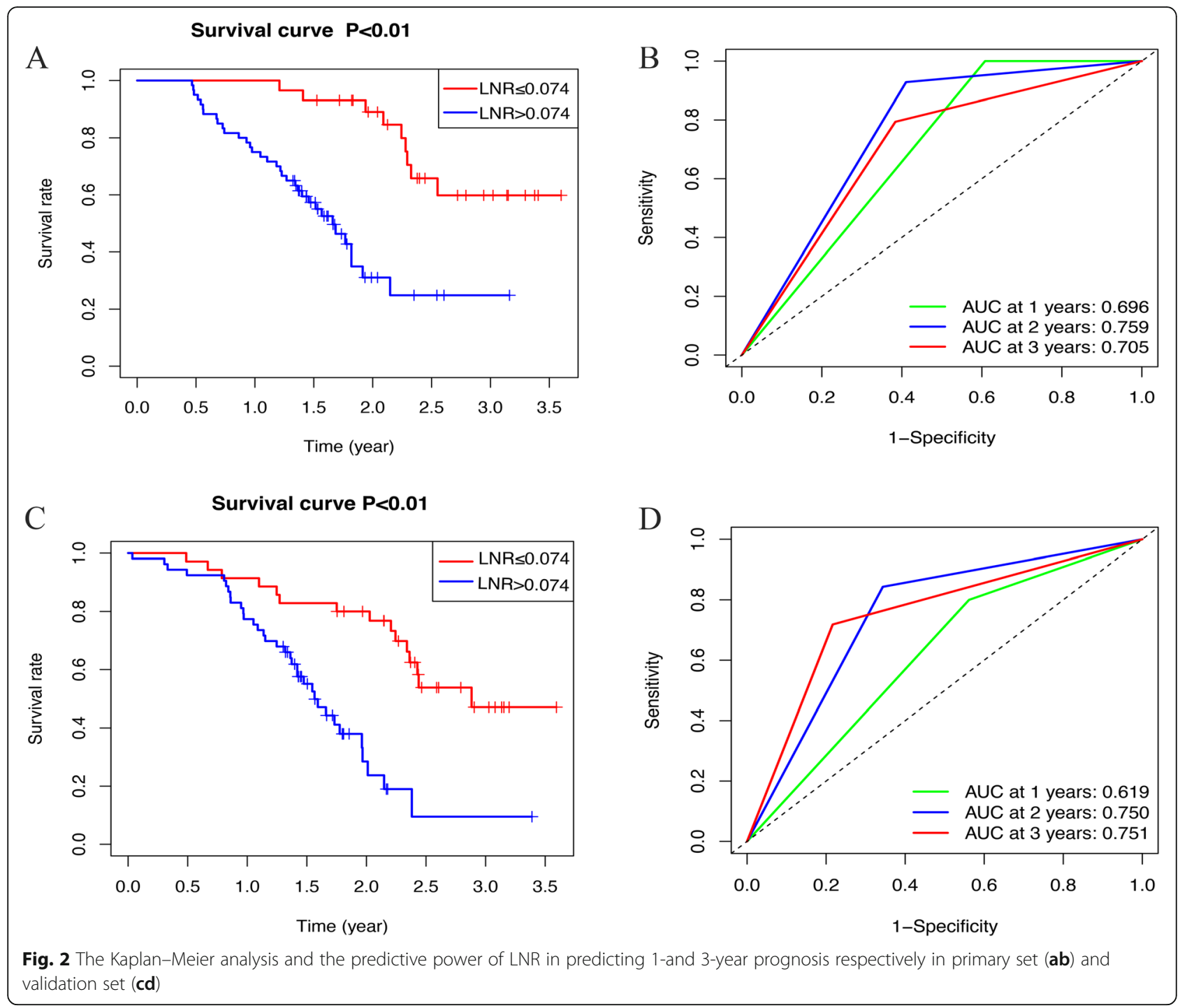

study: pathologically confirmed not to have adenocarcinoma $(n=79)$, adenocarcinoma of the pancreatic head with distant metastasis $(n=23)$ or lost to follow-up after not more than 1 month $(n=37)$. Adenocarcinoma of the pancreatic head patients underwent pancreaticoduodenectomy and systematic lymphadenectomy without peritoneal dissemination or distant metastases. Finally, 177 patients with adenocarcinoma of the pancreatic head were incorporated in the study. For further analysis, the discerned patients were randomly divided into a primary set $(n=89)$ and a validation set $(n=88)$. The follow-up time was more than 3 years.

\section{Data collection}

In resectable adenocarcinoma of the pancreatic head patients, related characteristics, such as age, sex, serum albumin, total lymphocyte count, initial serum level of carcinoembryonic antigen (CEA), preoperative carbohydrate antigen 19-9 (CA19-9), postoperative adjuvant systemic chemotherapy, and TNM stage, were screened through the electronic medical records. The PNI value was calculated as $10 \times$ serum albumin $(\mathrm{g} / \mathrm{dL})+0.005 \times$ total lymphocyte count $(/ \mathrm{mm} 3)$ in peripheral blood [17]. In accordance with the American Joint Committee on Cancer (AJCC) 8th edition guidelines, resectable pancreatic head cancer patients were sorted into diverse stages. OS was delimited as the phase from the time of diagnosis until death.

\section{Statistical analysis}

Survival distributions were estimated by the KaplanMeier method and log-rank test to compare the categorical variables of the primary set and validation set. Univariate and multivariate analyses were 


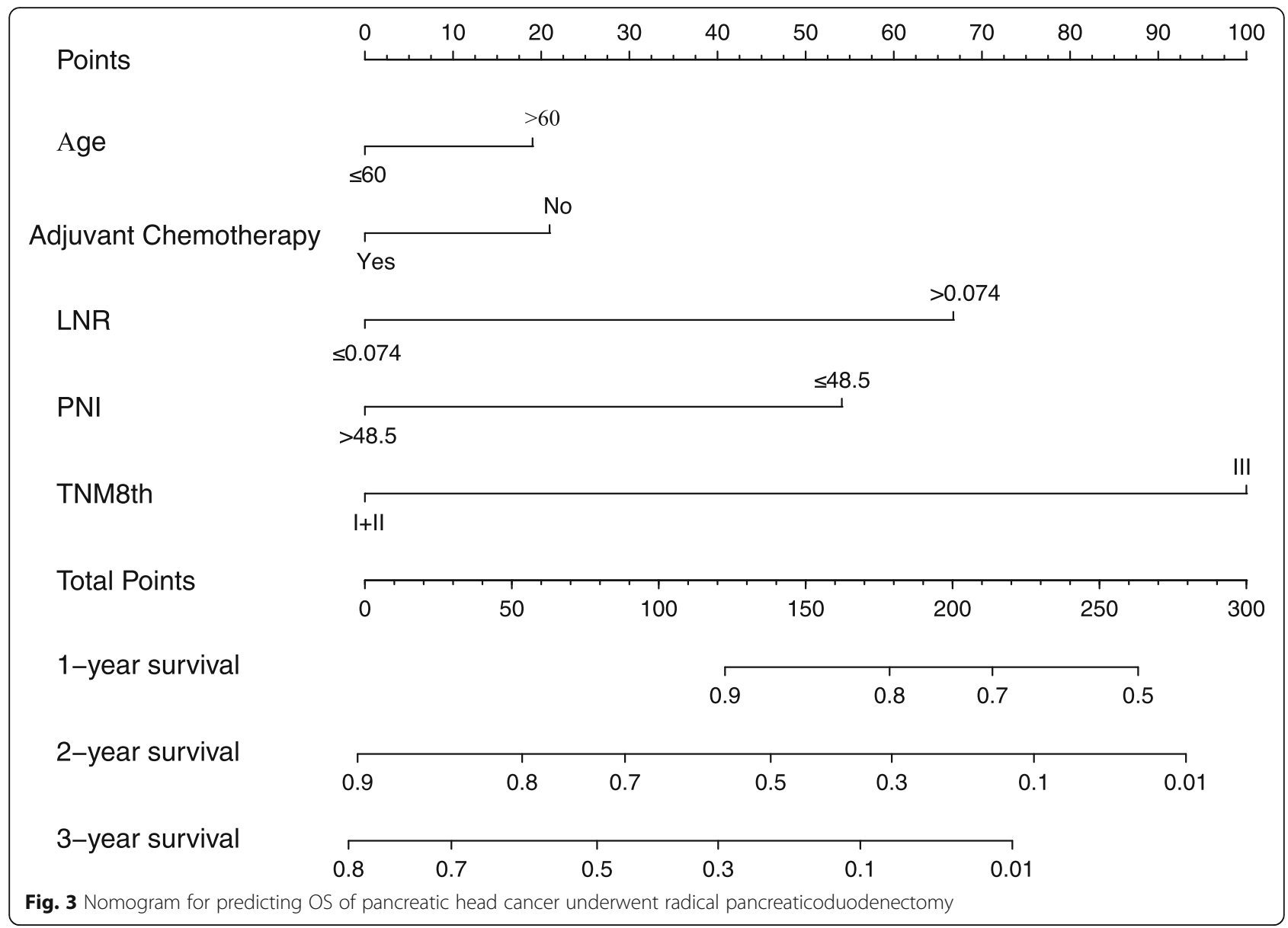

performed by using the Cox proportional hazards regression model. The multivariate analysis results were implemented to construct a nomogram. The concordance index (c-index), the area under the curve (AUC) and calibration were utilized to evaluate the predictive performance of the nomogram. Decision curve analysis (DCA) was implemented to evaluate the predictive power of the nomogram. A value of $P<0.05$ was considered statistically significant. Statistical analyses were performed using SPSS V26.0 (SPSS Inc.) and R software v4.0.2 (R Foundation for Statistical Computing, Vienna, Austria).

\section{Results}

\section{Patient characteristics}

The clinical characteristics of adenocarcinoma of the pancreatic head patients who underwent radical pancreaticoduodenectomy are shown in supplement 1. A total of $61.5 \%$ of the patients were male. The CA19-9 level was elevated in $82.5 \%$ of pancreatic head cancer patients, and $65.5 \%$ of pancreatic head cancer patients who underwent radical pancreaticoduodenectomy had grade I/II disease according to the AJCC 8th edition guidelines. Approximately half of the pancreatic head cancer patients had elevated CEA levels at diagnosis.

\section{Analysis of risk factors for pancreatic head cancer}

The univariate analysis and the multivariate analysis showed that the PNI, LNR, and TNM 8th edition guidelines were associated with $O S$ in the primary set (Table 1 ) and in the validation set (Table 2). Namely, the multivariate analysis revealed that the PNI (HR 0.51; 95\% CI, 0.273$0.952, P=0.034$ ), LNR (HR 2.543; 95\% CI, $1.052-$ 6.148, $P=0.038)$, and TNM 8th edition guidelines (HR 1.948; 95\% CI, 1.351-2.810, $P<0.001$ ) were independent factors related to $O S$ in the primary set. Similarly, the PNI (HR 0.398; 95\% CI, $0.217-$ $0.729, P=0.003$ ), LNR (HR 4.087; 95\% CI, 2.0658.090, $P<0.001)$, and TNM 8th edition guidelines (HR 2.786; 95\% CI, 1.939-4.003, $P<0.001$ ) performed by the multivariate analysis also remained independent variables related to $\mathrm{OS}$ in the validation set. 


\section{Construction of the nomogram}

The Kaplan-Meier analysis of the PNI in the primary set (Fig. 1a) and validation set (Fig. 1c) was statistically significant $(P<0.01)$. The AUC values for evaluating the performance of the PNI for 1-, 2 -, and 3-year OS were $0.855,0.875$ and 0.862 , respectively, in the primary set (Fig. 1b). The AUC values for evaluating the performance of the PNI for 1-, 2 -, and 3-year OS were $0.739,0.922$ and 0.874 , respectively, in the validation set (Fig. 1d). Additionally, the performance of the LNR for 3-year OS was 0.705 in the primary set and 0.751 in the validation set (Fig. 2). As age and postoperative adjuvant systemic chemotherapy are always regarded as predictive factors associated with pancreatic cancer prognosis, these factors were integrated into the nomogram [18-22]. The factors of age, postoperative adjuvant systemic chemotherapy, the PNI, the LNR, and the TNM 8th edition guidelines were implemented to construct a nomogram (Fig. 3).

\section{Comparison and validation of the nomogram}

The c-indexes of the nomogram were 0.799 (confidence interval (CI), 0.741-0.858) and $0.732(0.657-0.807)$ in the primary set and validation set, respectively. The calibration plot for predicting 1-, 2-, and 3-year OS (Fig. 4) showed that the nomogram model performed well in the primary set and validation set. The AUCs of the nomogram at 1 and 3 years were 0.832 and 0.783 , respectively, which were superior to the AJCC staging values of 0.759 and 0.705 (Fig. 5). Additionally, our study suggests that the nomogram showed a superior net benefit across a wider scale of threshold probabilities for predicting OS in the DCA (Fig. 6).

\section{Discussion}

We retrospectively collected the clinical characteristics of 316 patients with supposed pancreatic head cancer who were admitted to the West China Hospital of Sichuan University from July 2015 to June 2017. Finally,

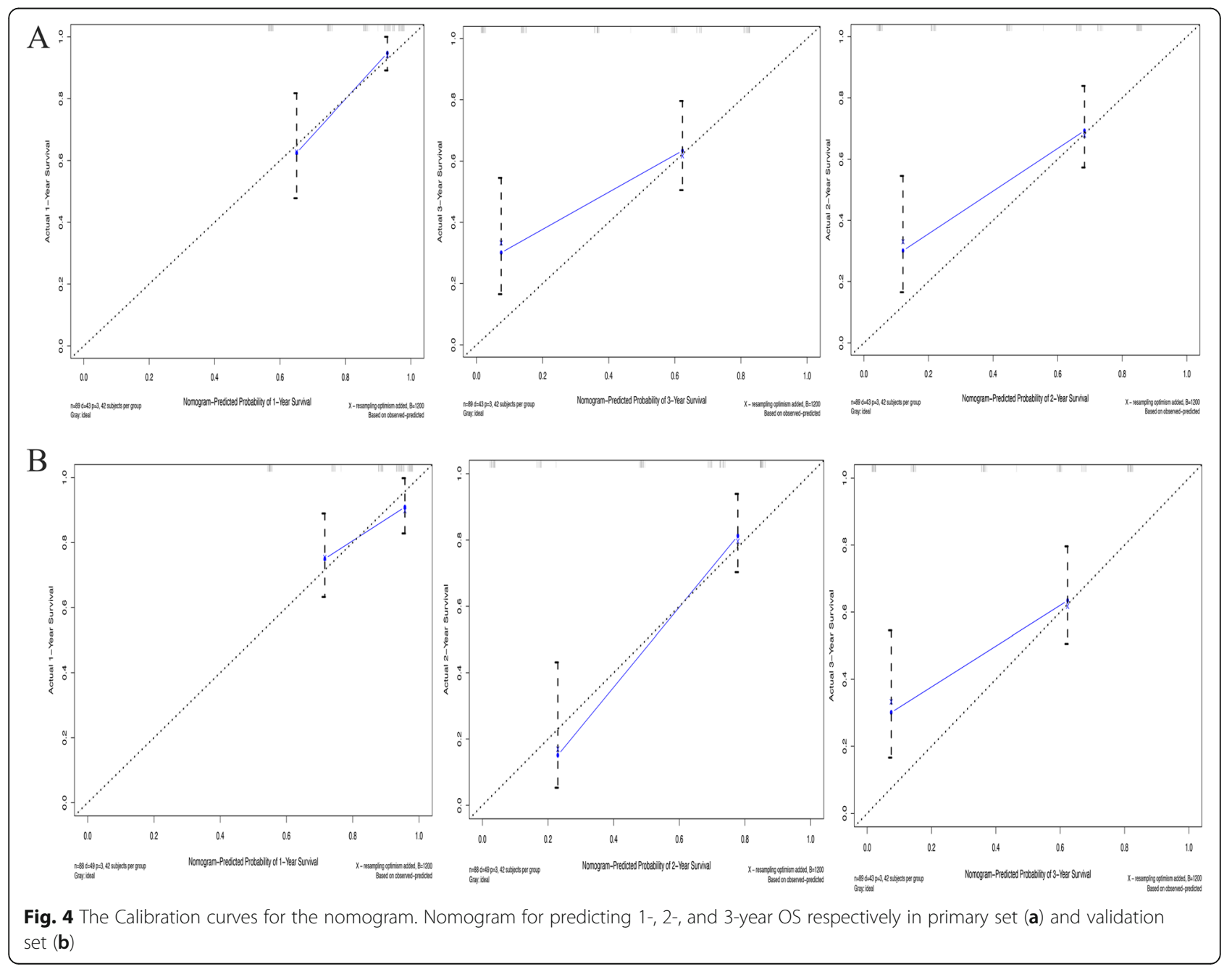



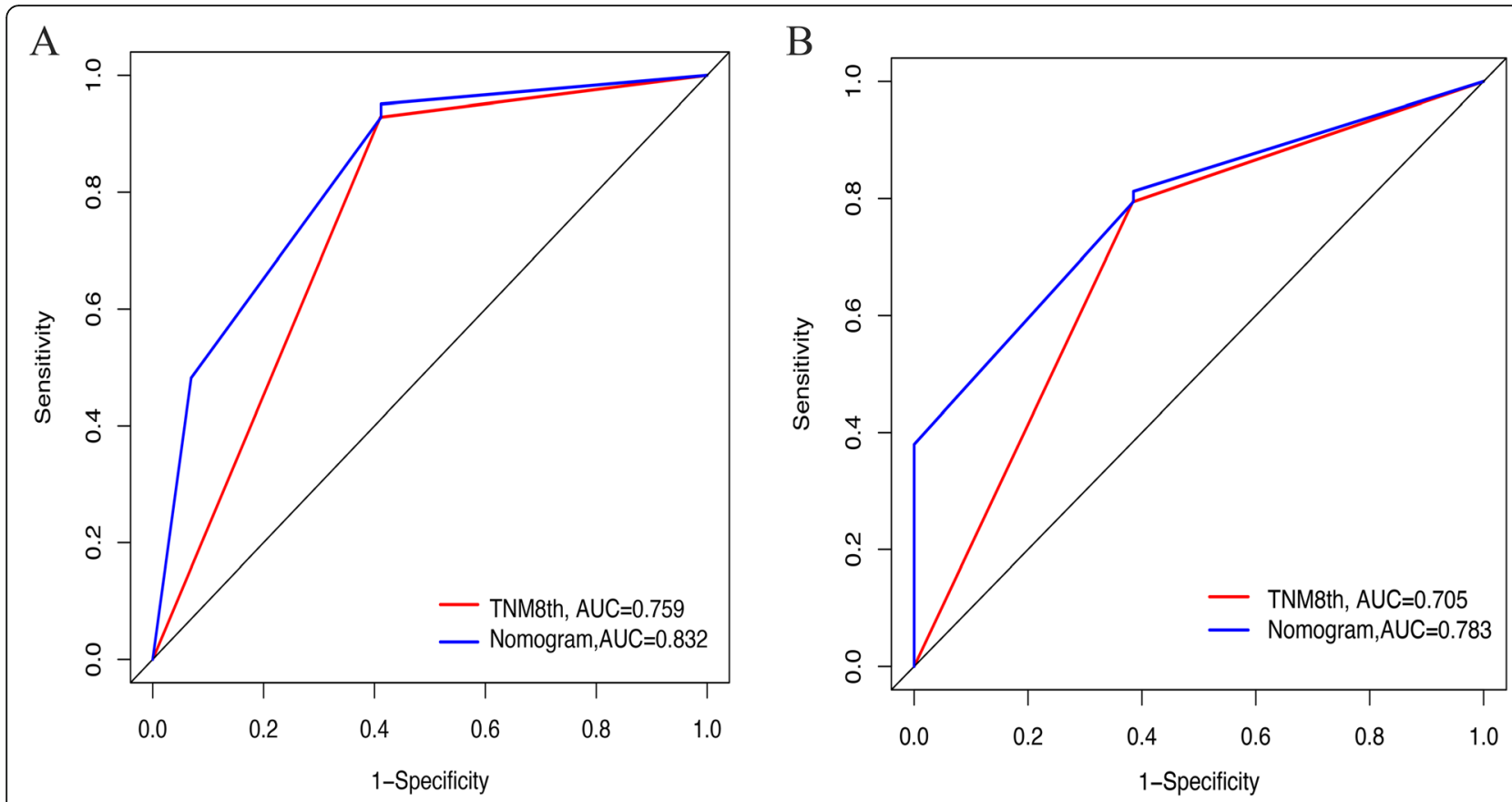

Fig. 5 Comparison of the performance of the nomogram and AJCC stage by AUC at 1 and 3 years in primary set (a) and validation set (b)

177 adenocarcinoma of the pancreatic head patients who underwent radical pancreaticoduodenectomy and systematic lymphadenectomy without peritoneal dissemination or distant metastases were included in the study. Subsequently, we screened out risk factors associated with the prognosis of head cancer patients who underwent pancreaticoduodenectomy to construct a nomogram. The calibration curves of the nomogram for predicting 1- and 2-year OS closely matched the ideal 45-degree line in the primary set (Fig. 4a) and validation set (Fig. 4b), meaning that the predictive power of the nomogram was significantly good. Although the calibration curves of the nomogram for predicting 3-year OS slightly deviated from the ideal 45-degree line in the primary set and validation set, notably, the c-indexes of the nomogram were $0.799(0.741-0.858)$ and $0.732(0.657-$ 0.807 ) in the primary set and validation set, respectively. Additionally, the AUCs of the nomogram at 1 and 3 years were 0.832 and 0.783 , respectively, which were superior to the AJCC staging values of 0.759 and 0.705 (Fig. 5). Finally, the nomogram showed a superior net benefit across a wider scale of threshold probabilities for predicting OS in the DCA. Thus, the nomogram may be an effective model for developing an optimal therapeutic schedule for adenocarcinoma of the pancreatic head patients.

Accumulating studies have been utilized to disclose the relationship between clinical characteristics and prognostic outcomes in pancreatic adenocarcinoma. For instance, lymph node metastases are considered an important factor for predicting OS in pancreatic cancer patients who undergo surgery [23-26]. However, previous studies have revealed that there are some limitations of only using the number of positive LNs to predict prognosis. The LNR is the ratio of the number of positive lymph nodes to the total number of lymph nodes dissected during surgery. In this study, the multivariate analysis suggested that the LNR was an independent factor related to OS. Additionally, previous studies have revealed that the PNI has been used as a predictive prognostic factor for hepatocellular carcinoma [27, 28], small-cell lung cancer [29, 30], nasopharyngeal carcinoma [31], gastric cancer [32], and pancreatic cancer [10]. Our study also suggested that the PNI was an independent factor for predicting OS in pancreatic head cancer patients who underwent pancreaticoduodenectomy. The published data revealed that preoperative enteral alimentation increased the serum albumin level and total lymphocyte count to improve postoperative outcomes, showing the indispensability of perioperative nutritional management [33-35]. For this reason, malnourished patients need early effective nutritional intervention to promote the treatment efficacy of resectable pancreatic head cancer. Collectively, the results of the nomogram constructed by the factors of age, postoperative adjuvant systemic chemotherapy, the PNI, and the LNR may serve 

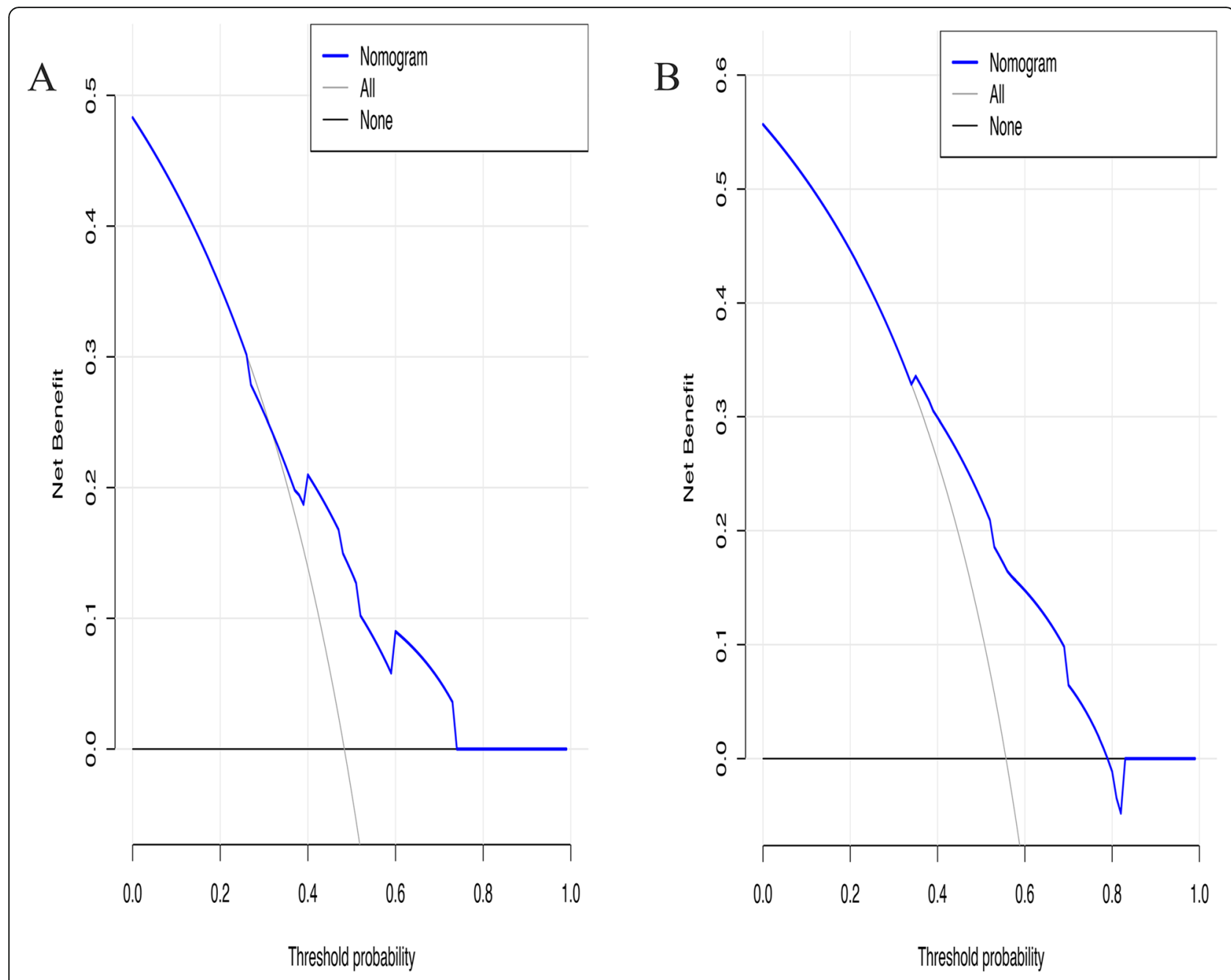

Fig. 6 Decision curve analysis of pancreatic head cancer underwent radical pancreaticoduodenectomy in primary set (a) and validation set (b). The horizontal solid black line assumed no patients would die, and the solid grey line assumed all patients would die

as a proposal of prognostic importance for adenocarcinoma of the pancreatic head patients.

There are limitations to this study. There is a lack of sufficient predictive factor incorporation in the nomogram to provide absolute predictions. Some known factors may not have been incorporated due to the absence of numbers or observations, or there may be biomarkers that are still undisclosed. This study only used a single institution-based database; therefore, to verify the accuracy of predictive nomograms in multiple institutionbased databases is necessary.

Conclusions: The nomogram may be used to predict the prognosis of radical resection for adenocarcinoma of the pancreatic head. These findings may represent an effective model for developing an optimal therapeutic schedule for malnourished patients who need early effective nutritional intervention and may promote the treatment efficacy of resectable adenocarcinoma of the pancreatic head.

\section{Abbreviations}

AUC: Area under the curve; PNI: Prognostic nutritional index; LNR: Lymph node ratio; AJCC: American Joint Committee on Cancer; Cl: Confidence interval; OS: overall survival

\section{Supplementary Information}

The online version contains supplementary material available at https://doi. org/10.1186/s12885-021-08295-5.

Additional file 1. The demographics of set.

\section{Acknowledgements}

The authors would like to thank the colleagues who made this work possible.

\section{Authors' contributions}

CW, ZWW, SZH, XH and ZIW participated in the drafting, writing, and revising of the manuscript. Revising of the manuscript. BLT participated in the conception and design of the study. Both of them contributed to analysis and interpretation of the data and they approved the final version of the manuscript to be submitted, and agreed to be accountable for all aspects of the work. All authors read and approved the final manuscript. 


\section{Funding}

No funding was received for this study.

\section{Availability of data and materials}

All primary data is available by sending email to correspondence author.

\section{Declarations}

\section{Ethics approval and consent to participate}

The clinical data was acquired with the approval and permission of the West China Hospital Review Board. The study protocol was approved by the Ethical Review Committees of Sichuan University. Informed consent was not required because this study was a retrospective report of cases, which is a retrospective analysis of clinical data with no relevant to human biological ethic problems.

\section{Consent for publication}

The authors have seen the manuscript and approved to submit to your journal.

\section{Competing interests}

The authors declare that they have no conflict of interest.

Received: 19 November 2020 Accepted: 5 May 2021

Published online: 27 May 2021

\section{References}

1. Bray F, Ferlay J, Soerjomataram I, Siegel RL, Torre LA, Jemal A. Global cancer statistics 2018: GLOBOCAN estimates of incidence and mortality worldwide for 36 cancers in 185 countries. CA Cancer J Clin. 2018;68(6):394-424. https://doi.org/10.3322/caac.21492.

2. Kamisawa T, Wood LD, Itoi T, Takaori K. Pancreatic cancer. Lancet. 2016; 388(10039):73-85. https://doi.org/10.1016/S0140-6736(16)00141-0.

3. Strobel O, Neoptolemos J, Jaeger D, Buechler MW. Optimizing the outcomes of pancreatic cancer surgery. Nat Rev Clin Oncol. 2019;16(1):1126. https://doi.org/10.1038/s41571-018-0112-1.

4. Elshaer M, Gravante G, Kosmin M, Riaz A, Al-Bahrani A. A systematic review of the prognostic value of lymph node ratio, number of positive nodes and total nodes examined in pancreatic ductal adenocarcinoma. Ann R Coll Surg Engl. 2017;99(2):101-6. https://doi.org/10.1308/rcsann.2016.0340.

5. Morimoto D, Yamada S, Murotani K, Sonohara F, Takami H, Suenaga M, et al. Prognostic impact of portal system invasion in pancreatic cancer based on image classification. Pancreas. 2018:47(10):1350-6. https://doi.org/10.1097/ MPA.0000000000001186

6. Wasif N, Ko CY, Farrell J, Wainberg Z, Hines OJ, Reber H, et al. Impact of tumor grade on prognosis in pancreatic cancer: should we include grade in AJCC staging? Ann Surg Oncol. 2010;17(9):2312-20. https://doi.org/10.1245/ s10434-010-1071-7.

7. Lee SH, Chung MJ, Kim B, Lee HS, Lee HJ, Heo JY, et al. The significance of the prognostic nutritional index for all stages of pancreatic cancer. Nutr Cancer. 2017;69(3):512-9. https://doi.org/10.1080/01635581.2016.1250921.

8. La Torre M, Nigri G, Petrucciani N, Cavallini M, Aurello P, Cosenza G, et al. Prognostic assessment of different lymph node staging methods for pancreatic cancer with RO resection: pN staging, lymph node ratio, log odds of positive lymph nodes. Pancreatology. 2014;14(4):289-94. https://doi.org/1 0.1016/j.pan.2014.05.794.

9. Onodera T, Goseki N, Kosaki G. Prognostic nutritional index in gastrointestinal surgery of malnourished cancer patients. Nihon Geka Gakkai Zasshi. 1984:85(9):1001-5.

10. Kanda M, Fujii T, Kodera Y, Nagai S, Takeda S, Nakao A. Nutritional predictors of postoperative outcome in pancreatic cancer. Br J Surg. 2011;98(2):268-74. https://doi.org/10.1002/bjs.7305.

11. You MS, Lee SH, Choi YH, Shin B-S, Paik WH, Ryu JK, et al. Lymph node ratio as valuable predictor in pancreatic cancer treated with R0 resection and adjuvant treatment. BMC Cancer. 2019;19(1):952. https://doi.org/10.11 86/s12885-019-6193-0.

12. Aoyama T, Yamamoto N, Kamiya M, Murakawa M, Tamagawa H, Sawazaki S, et al. The lymph node ratio is an independent prognostic factor in pancreatic cancer patients who receive curative resection followed by adjuvant chemotherapy. Anticancer Res. 2018;38(8):4877-82. https://doi. org/10.21873/anticanres.12801.
13. Robinson S, Rahman A, Haugk B, French J, Manas D, Jaques B, et al. Metastatic lymph node ratio as an important prognostic factor in pancreatic ductal adenocarcinoma. EJSO. 2012;38(4):333-9. https://doi.org/10.1016/j. ejso.2011.12.020.

14. Murakami Y, Uemura K, Sudo T, Hayashidani Y, Hashimoto Y, Nakashima A, et al. Number of metastatic lymph nodes, but not lymph node ratio, is an independent prognostic factor after resection of pancreatic carcinoma. J Am Coll Surg. 2010;211(2):196-204. https://doi.org/10.1016/j.jamcollsurg.201 0.03 .037$.

15. Yamamoto $Y$, Ikoma H, Morimura R, Konishi $H$, Murayama $Y$, Komatsu $S$, et al. The clinical impact of the lymph node ratio as a prognostic factor after resection of pancreatic cancer. Anticancer Res. 2014;34(5):2389-94.

16. Liu Z-Q, Xiao Z-W, Luo G-P, Liu L, Liu C, Xu J, et al. Effect of the number of positive lymph nodes and lymph node ratio on prognosis of patients after resection of pancreatic adenocarcinoma. Hepatobiliary Pancreat Dis Int. 2014;13(6):634-41. https://doi.org/10.1016/S1499-3872(14)60264-2.

17. Schiesser M, Kirchhoff P, Müller MK, Schäfer M, Clavien P-A. The correlation of nutrition risk index, nutrition risk score, and bioimpedance analysis with postoperative complications in patients undergoing gastrointestinal surgery. Surgery. 2009;145(5):519-26. https://doi.org/10.1016/j.surg.2009.02.001.

18. Altman AM, Wirth K, Marmor S, Lou E, Chang K, Hui JY, et al. Completion of adjuvant chemotherapy after upfront surgical resection for pancreatic cancer is uncommon yet associated with improved survival. Ann Surg Oncol. 2019;26(12):4108-16. https://doi.org/10.1245/s10434-019-07602-6.

19. White RJ, Hasan S, Monga D, Finley G, Islam M, Schiffman S, et al. Time to adjuvant systemic therapy following pancreatic cancer resection and effect on outcome. Pancreas. 2019;48(8):1086-91. https://doi.org/10.1097/MPA. 0000000000001373

20. Wang H, Liu J, Xia G, Lei S, Huang X, Huang X. Survival of pancreatic cancer patients is negatively correlated with age at diagnosis: a population-based retrospective study. Sci Rep. 2020;10(1):1-9.

21. Bagni $K$, Chen IM, Johansen AZ, Dehlendorff $C$, Jensen BV, Hansen $C P$, et al. Prognostic impact of Charlson's age-comorbidity index and other risk factors in patients with pancreatic cancer. Eur J Cancer Care. 2020;29(3): e13219. https://doi.org/10.1111/ecc.13219.

22. Iwai N, Okuda T, Sakagami J, Harada T, Ohara T, Taniguchi M, et al. Neutrophil to lymphocyte ratio predicts prognosis in unresectable pancreatic cancer. Sci Rep. 2020;10(1):1-7.

23. Brennan MF, Kattan MW, Klimstra D, Conlon K. Prognostic nomogram for patients undergoing resection for adenocarcinoma of the pancreas. Ann Surg. 2004;240(2):293-8. https://doi.org/10.1097/01.sla.0000133125.85489.07.

24. Bian Y, Guo S, Jiang H, Gao S, Shao C, Cao K, et al. Relationship between radiomics and risk of lymph node metastasis in pancreatic ductal adenocarcinoma. Pancreas. 2019;48(9):1195-203. https://doi.org/10.1097/ MPA.0000000000001404

25. Suzuki Y, Takadate T, Mizuma M, Shima H, Suzuki T, Tachibana T, et al. Stromal expression of hemopexin is associated with lymph-node metastasis in pancreatic ductal adenocarcinoma. PLoS One. 2020;15(7):e0235904. https://doi.org/10.1371/journal.pone.0235904.

26. Collard M, Voron T, Svrcek M, Ahmed O, Balladur P, Hor T, et al. Para-aortic lymph node metastasis detected intraoperatively by systematic frozen section examination in pancreatic head adenocarcinoma: is resection improving the prognosis? HPB. 2020;22(11):1604-12.

27. Pinato D, North B, Sharma R. A novel, externally validated inflammationbased prognostic algorithm in hepatocellular carcinoma: the prognostic nutritional index (PNI). Br J Cancer. 2012;106(8):1439-45. https://doi.org/10.1 038/bjc.2012.92

28. Chan AW, Chan SL, Wong GL, Wong WW, Chong CC, Lai PB, et al. Prognostic nutritional index (PNI) predicts tumor recurrence of very early/early stage hepatocellular carcinoma after surgical resection. Ann Surg Oncol. 2015; 22(13):4138-48. https://doi.org/10.1245/s10434-015-4516-1.

29. Hong S, Zhou T, Fang W, Xue C, Hu Z, Qin T, et al. The prognostic nutritional index (PNI) predicts overall survival of small-cell lung cancer patients. Tumor Biol. 2015;36(5):3389-97. https://doi.org/10.1007/s13277014-2973-y.

30. Jin S, Cao S, Xu S, Wang C, Meng Q, Yu Y. Clinical impact of pretreatment prognostic nutritional index (PNI) in small cell lung cancer patients treated with platinum-based chemotherapy. Clin Respir J. 2018;12(9):2433-40. https://doi.org/10.1111/crj.12925.

31. Yang L, Xia L, Wang Y, Hong $S$, Chen $H$, Liang $S$, et al. Low prognostic nutritional index (PNI) predicts unfavorable distant metastasis-free survival in 
nasopharyngeal carcinoma: a propensity score-matched analysis. PLoS One. 2016;11(7):e0158853. https://doi.org/10.1371/journal.pone.0158853.

32. Migita K, Takayama T, Saeki K, Matsumoto S, Wakatsuki K, Enomoto K, et al. The prognostic nutritional index predicts long-term outcomes of gastric cancer patients independent of tumor stage. Ann Surg Oncol. 2013;20(8): 2647-54. https://doi.org/10.1245/s10434-013-2926-5.

33. Xu J, Zhong Y, Jing D, Wu Z. Preoperative enteral immunonutrition improves postoperative outcome in patients with gastrointestinal cancer. World J Surg. 2006;30(7):1284-9. https://doi.org/10.1007/s00268-005-0756-8.

34. Fukuda T, Seto Y, Yamada K, Hiki N, Fukunaga T, Oyama S, et al. Can immune-enhancing nutrients reduce postoperative complications in patients undergoing esophageal surgery? Dis Esophagus. 2008;21(8):708-11. https://doi.org/10.1111/j.1442-2050.2008.00861.x

35. Zaloga GP. Parenteral nutrition in adult inpatients with functioning gastrointestinal tracts: assessment of outcomes. Lancet. 2006;367(9516): 1101-11. https://doi.org/10.1016/50140-6736(06)68307-4.

\section{Publisher's Note}

Springer Nature remains neutral with regard to jurisdictional claims in published maps and institutional affiliations.

Ready to submit your research? Choose BMC and benefit from:

- fast, convenient online submission

- thorough peer review by experienced researchers in your field

- rapid publication on acceptance

- support for research data, including large and complex data types

- gold Open Access which fosters wider collaboration and increased citations

- maximum visibility for your research: over $100 \mathrm{M}$ website views per year

At BMC, research is always in progress.

Learn more biomedcentral.com/submissions 